\title{
Enhancement of optical absorption in Ga-chalcopyrite-based intermediate-band materials for high efficiency solar cells
}

\author{
I. Aguilera *, P. Palacios, P. Wahnón \\ Instituto de Energia Solar and Departamento Tecnoiogias Especiales, ETSI Telecomunicación, UPM, Ciudad Universitaria, Madrid 28040. Spain
}

\section{A R T I C L E I N F O}

Keywords:

Chalcopyrites

Intermediate band

Optical properties

\begin{abstract}
A B S T R A C T
We present absorption properties enhancement for two $\mathrm{CuGaS}_{2}$-based intermediate-band materials, as promising compounds for high efficiency, lower-cost photovoltaic devices. Previous band diagrams calculations predicted that these materials present a partially filled localized band within the band gap of the host semiconductor, which would increase the absorption of low-energy photons, creating additional electron-hole pairs respect to a conventional semiconductor. This could ideally result in an increase of the photocurrent of the cell without the fall of the open-circuit voltage. In this paper we show, using density functional methods, the effect of this intermediate band on the optical properties of the derived alloys. We highlight the significant enhancement of the absorption coefficient observed in the most intense range of the solar emission and we study the reflectance and transmittance properties of the materials in order to understand the effect of the thickness of the sample on the optical properties. We compare two different substituents of the $\mathrm{Ga}_{\text {atoms in }} \mathrm{CuGaS}_{2}$, namely. Ti and $\mathrm{Cr}$ atoms, able to form the intermediate-band material, and their interest for photovoltaic applications.
\end{abstract}

\section{Introduction}

Very recently, we have presented studies of ab initio optical properties of the spinel semiconductor $\left[n_{2} S_{3}\right.$ substituted with transition metals, such as $\mathrm{V}$ and $\mathrm{Ti}[1 \mid$. Those compounds were proposed due to the possibility of the $3 d$ electrons of the transition metals of forming a partially filled narrow band isolated from the valence and the conduction band of the host semiconductor. This particular electronic structure allows the absorption of sub-band-gap energy photons that would result, in a solar cell, in the creation of additional electron-hole pairs and in the increase of the photocurrent without the fall of the open-circuit voltage. A cell based on such material could reach theoretical efficiencies up to $63.2 \%$ [2].

Later, due to our promising predictions, the $V$-substituted $\ln _{2} S_{3}$ was synthesized by hydrothermal method, being the first time that a partially filled intermediate-band material, absorbing across the full solar spectrum range, was synthesized. Its optical properties were later measured, finding very good agreement with our theoretical results [3], thus confirming the prediction capabilities of our methodology.

Following the same method within the chalcopyrite framework, starting from the semiconductor $\mathrm{CuGaS}_{2}$, our group proposed, for enhanced photovoltaic applications, replacing a fraction of the $\mathrm{Ga}$ atoms by $\mathrm{Ti}$ or $\mathrm{Cr}$ (at tetrahedral sites) [4-6].
There have also been proposals of transition-metal substitution in $\mathrm{CuGaS}_{2}$ for other uses, such as Mn-doped $\mathrm{CuGaS}_{2}$, which was theoretically predicted by $a b$ initio methods $[7,8]$ and later synthesized by chemical vapor transport [9․

Nowadays, chalcopyrite-type semiconductors represent one of the most developed thin-films technologies in the search for more efficient, lower-cost solar cells. As a consequence of the interest on these semiconductors, many of their properties are well known and several experimenta] [10-12] and theoretical $[13,14]$ data are available, thus, making them a solid base for this study. Among the chalcopyrites, we proposed $\mathrm{CuGaS}_{2}$ as the best host since its band gap is wide enough (2.4-2.5 eV [15-17]) to accommodate an isolated narrow intermediate band for photovoltaic applications.

The main purpose of the present work is to compare in detail the two most promising transition-metal substituents ( $\mathrm{Ti}, \mathrm{Cr}$ ) of $\mathrm{Ga}$ in $\mathrm{CuGaS}_{2}$ by means of the optical properties of the resulting compounds. These transition metals were chosen by electronic considerations in recent works [4-6], but as the optical behavior is a deciding factor in the quest of an efficient photovoltaic material, a deeper study and interpretation of optical properties of intermediate-band compounds is needed.

\section{Method}

In this work, optical absorption and dielectric properties of Ti- and $\mathrm{Cr}$-substituted $\mathrm{CuGaS}_{2}$ host semiconductor are obtained by $a b$ initio calculations. Both studied alloys have a transition-metal 
concentration level of $12.5 \%$, with stoichiometry $\mathrm{Cu}_{4} \mathrm{MGa}_{3} \mathrm{~S}_{8}$ $(\mathrm{M}=\mathrm{Ti}, \mathrm{Cr})$. For the ground state, the cells were fully structurally and electronically relaxed [4], since the structural parameters are critical in chalcopyrites [13], playing an important role on the electronic properties of these compounds. Cell geometry, lattice parameters, and internal anjon distortion, were determined theoretically by minimization of the forces and the total energy, finding good agreement both with experimental and other theoretical results of the host semiconductor. The tolerance for atomic forces in both relaxations was $0.01 \mathrm{eV} / \mathrm{A}$.

The electronic ground state of each material was calculated with the plane-wave code vasp [18-20], using spin-polarized density functional theory (DFT) in the generalized gradient approximation (GGA) with the Perdew-Wang 1991 functional [21].

We obtain the dielectric and optical properties using Fermi's golden rule, as the sum over independent transitions between Kohn-Sham states, as derived and described by Gajdos et al. [22] and implemented in the optic code, using projector augmented wave (PAW) pseudopotentials $[23,24 \mid$ and without local-field effects (i.e., neglecting the off-diagonal elements of the dielectric matrix). We have only considered interband and direct transitions for the determination of the imaginary part of the dielectric function. Since indirect transitions are a three particles process (thus, less probable), we expect the contribution of these transitions to be much lower than that of the direct ones.

The real part of the dielectric tensor was obtained from the imaginary part by the Kramers-Krönig relations. To get a converged frequency-dependent dielectric tensor, the Brillouin zone was sampled using a $12 \times 12 \times 12$ Monkhorst-Pack grid including the $\Gamma$-point and 160 empty bands were used.

The use of PAW pseudopotentials allows for a reduction of the number of plane-waves required for the calculations, giving the same accuracy, which is very significant in cases like ours with localized (d) electrons. The valence configurations used in this work were: $3 d^{10} 4 s^{1}$ for $\mathrm{Cu} ; 4 s^{2} 4 p^{1}$ for $\mathrm{Ga} ; 3 s^{2} 3 \mathrm{p}^{4}$ for $S ; 3 d^{2} 4 s^{2}$ for $\mathrm{Ti}$ and $3 \mathrm{~d}^{5} 4 \mathrm{~s}^{1}$ for $\mathrm{Cr}$.

As a reference to understand the optical spectra presented in the following sections, Fig. 1 shows the density of states (DOS) of the host semiconductor and the effect of the insertion of the transition metal. In order to stress the origin of the intermediate band, the atomic projection of the DOS is also shown.

Since it is well known that standard-DFT underestimates significantly the band gap of semiconductors, due to the inaccuracy in treating the excited states, it is necessary for the proper determination of the optical characteristics presented hereafter, to apply a correction to the excited states of the conduction band. In our case, we have found that the most reasonable correction is a simple shift of the conduction band eigenvalues to match the experimental band gap of the host semiconductor. This shift is justified, especially in the case of low concentrations of the transition metal where the intermediate band is not overlapping with the conduction band. We have carried out more accurate calculations, beyond DFT, within the exact exchange (EXX) approach $[25]$ and we have preliminary quasiparticles $\mathrm{CW}[26,27]$ results, and both have shown that the correction to the DFT problem can be indeed approximated by a rigid shift of the conduction band (see Ref. [28]).

\section{Results and discussion}

\subsection{Absorption}

Let us now focus on the total absorption coefficients obtained for the $\mathrm{Cu}_{4} \mathrm{MGa}_{3} \mathrm{~S}_{8}$ alloys. In Fig. 2, we show the absorption coefficient of $\mathrm{CuGaS}_{2}$ compared with the experimental

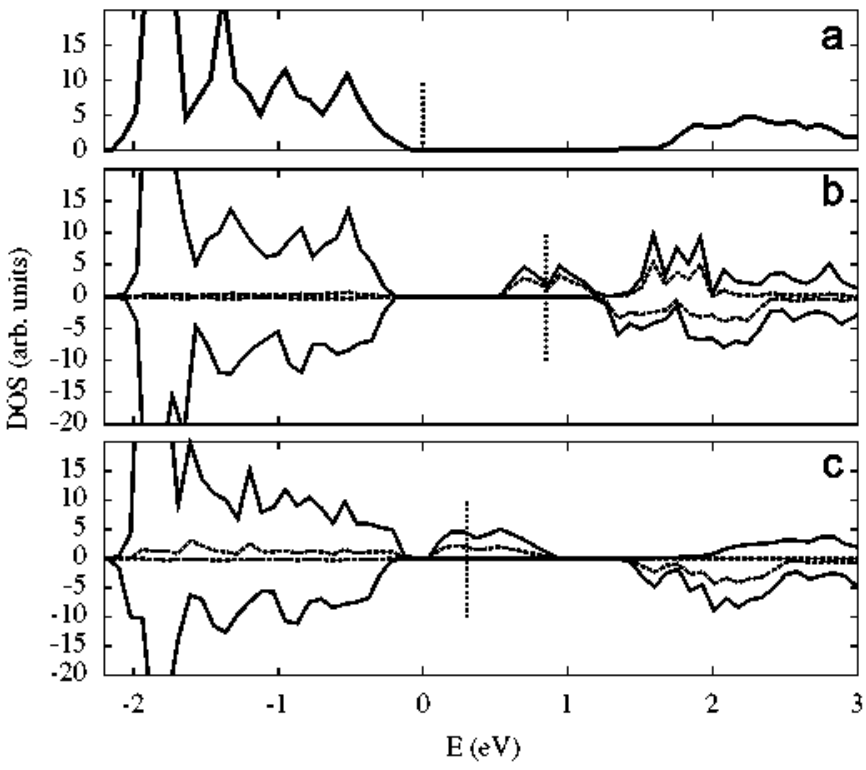

Fig. 1. (Color online) Total (solid line) densities of states (DOS) and transitionmetal projections (dotted line) of the (a) $\mathrm{CuGaS}_{2}$ semiconductor, (b) Ti-substituted alloy and (c) Cr-substituted compound (up and down spins). Vertical dotted lines indicate the Fermi energy.

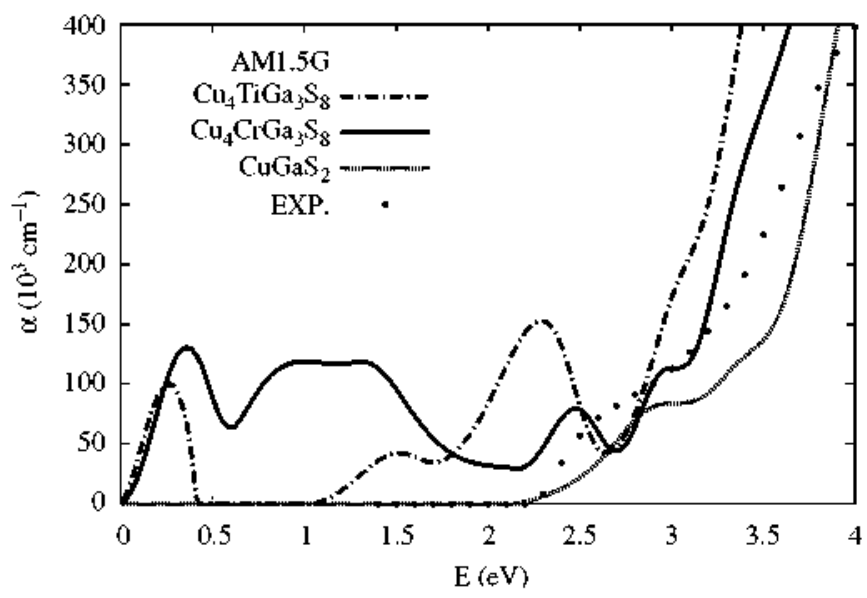

Fig. 2. (Color online) Absorption coefficient of the resulting intermediate-band compounds, compared to that of $\mathrm{CuGaS}_{2}$. As a reference: the solar spectrum, and experimental results of Ref. [10].

measurements by Alonso et al. [10] (this result was discussed in Ref. [29]), that is used here as a reference for the absorptions of the transition-metal alloys. The AM1.5G solar spectrum is shown in arbitrary units as a background to prove that the enhancement in the absorption due to the intermediate band appears mainly in the most intense part of the spectrum.

The position of the intermediate band inside the band gap becomes an important factor to be taken into consideration. In the case of the $\mathrm{Cr}$ as substituent, the intermediate band is very close to the valence band $(0.1 \mathrm{eV})$ [5], and that implies that the main increase in the absorption of sub-band-gap photons is found at around $0.5-1.5 \mathrm{eV}$ (Fig. 2), not coinciding with the maximum of the solar emission. Nevertheless, since other properties have to be taken into account, we do not rule out the $\mathrm{Cr}$ as a good candidate.

For the $\mathrm{Cu}_{4} \mathrm{TiGa}_{3} \mathrm{~S}_{8}$ compound, we know from previous works [4] that the intermediate band in this compound lies at about $0.7 \mathrm{eV}$ over the top of the valence band, which is close to the ideal 

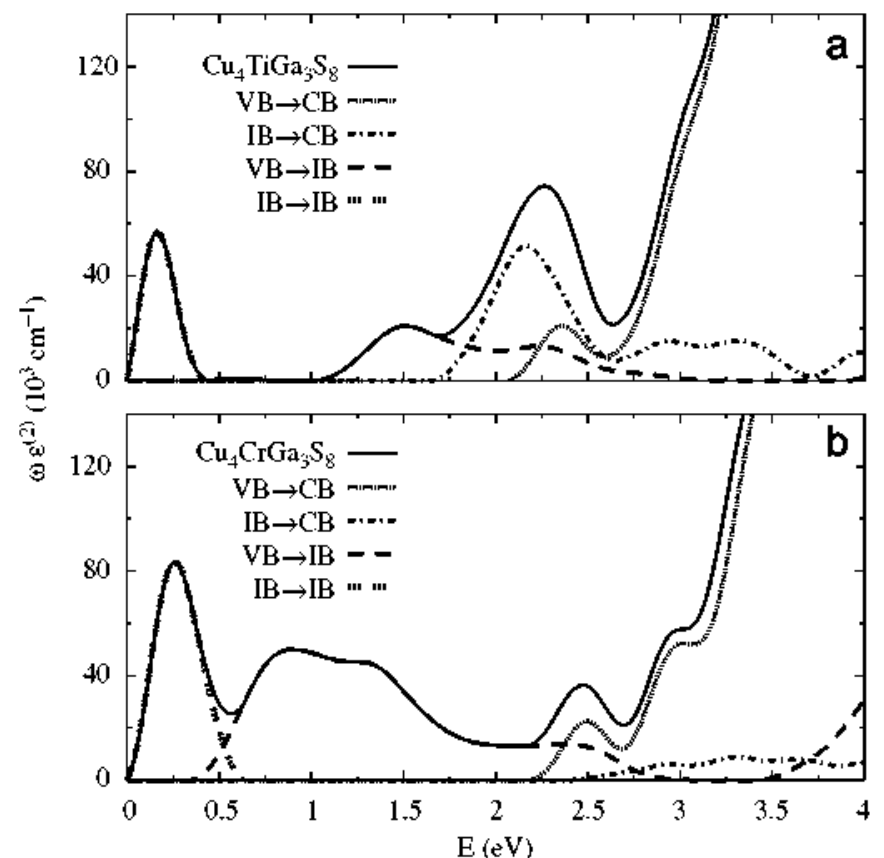

Fig. 3. Imaginary part of the dielectric function $\varepsilon^{(2)}$, multiplied by the frequency $\omega$, of the (a) $\mathrm{Cu}_{4} \mathrm{TiGa}_{3} \mathrm{~S}_{8}$ and (b) $\mathrm{Cu}_{4} \mathrm{CrGa}_{3} \mathrm{~S}_{8}$ intermediate-band materials (red-solid line). Partial contributions of the different characteristic transitions of these materials. (For interpretation of the references to color in this figure legend, the reader is referred to the web version of this article.)

situation [2]. In terms of the optical absorption this results in the new peaks lying in the main part of the solar spectrum (at 1.5 and $2.3 \mathrm{eV}$ ).

In order to reveal the precise effect of the partially filled transition-metal band on the optical spectra, we have carried out calculations to analyze the character of the transitions contributing to the main peaks of the spectra in Fig. 2. We explain those peaks by means of the imaginary part of the dielectric function of the two compounds.

The results for Ti substituting a $G$ a atom in the unit cell are shown in Fig. 3(a). For any intermediate-band material, the first peak in the absorption is due to transitions between the different states that form the intermediate band. These transitions, even though they contribute to the overall dynamic process of absorption, are not directly used in the generation of photocurrent in the cell, since one of the requirements of an intermediate band is to be isolated from the contacts of the cell and so the creation of electron-holes pairs in the intermediate band will not increase directly the photocurrent of the device.

Fig. 3(a) helps us understand all the major features in the spectrum of $\mathrm{Cu}_{4} \mathrm{TiGa}_{3} \mathrm{~S}_{8}$. We can now explain the character of the main peaks at 1.5 and $2.3 \mathrm{eV}$ seen in Fig. 2. The first one is principally due to transitions between the valence band and the intermediate band and thus having onset at around $0.7 \mathrm{eV}$. The principal peak, at $2.3 \mathrm{eV}$ is mainly due to transitions between the intermediate and the conduction band. The two peaks are at the energies of the higher solar emission, which was a region of forbidden absorption for the pure semiconductor.

In analogy with the interpretation for the Ti, results for the $\mathrm{Cr}$-alloy are shown in Fig. 3(b). The major low energy contribution in this case appears from around 0.5 to $1.5 \mathrm{eV}$, this broad peak is due to the wider intermediate band. The contribution of transitions from the intermediate to the conduction bands happens at energies higher than the solar emission, being not so relevant for the photovoltaic process. Besides, those transitions are less favored than those from the valence to the intermediate

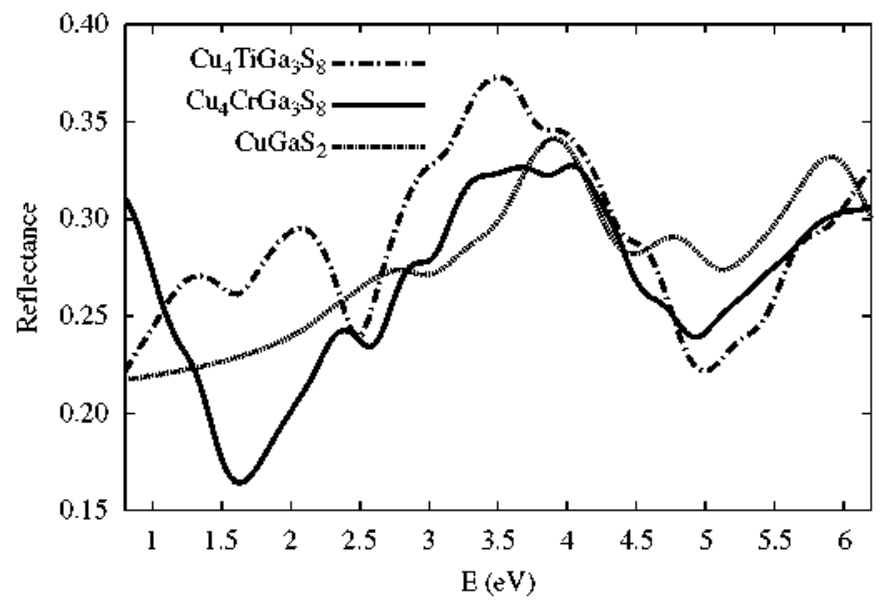

Fig. 4. (Color online) Reflectance of the $\mathrm{CuGaS}_{2}$ host semiconductor and the two intermediate-band materials.

band, while, in the ideal case, they should occur at approximately the same rate in order to contribute efficiently to the generation of electron-hole pairs with effect on the performance of the cell.

For both substituents we find a peak at around $2.5 \mathrm{eV}$ due to transitions between the valence band and the conduction band that did not appear in the absorption of the host semiconductor. This peak is due to transitions between the valence band and the bottom conduction, which, in the substituted alloys, have principally transition-metal d-states character (see projected DOS in Fig. 1), and therefore this peak is not present in the spectrum of the pure $\mathrm{CuGaS}_{2}$.

\subsection{Reflectance and transmittance}

In the quest of an efficient photovoltaic material, its reflectance plays also an important role. When combined with the absorption coefficient, it allows us to study, among other properties, refractive indexes, extinction coefficients, transmittances and absorptivities. The reflectance spectra in Fig. 4 show the very different behavior at low energies between the two substituents compared to the semiconductor, whereas from $2.5 \mathrm{eV}$ (approximately the gap of the host semiconductor) the three compounds present similar features. We consider this result can be interesting for the experimentalists with a view to the growth process of the intermediate-band alloys.

Making use of the reflectance $R$ and the absorption coefficient $\alpha$, the transmittance of the different compounds was studied, through relation (1), as a function of the thickness of the sample and the importance of this parameter was highlighted:

$T(\omega)=\frac{(1-R)^{2} e^{-\alpha w}}{1-R^{2} e^{-2 \alpha w}}$

The thicknesses $w$ were chosen in the range of typical widths of chalcopyrite absorbers in thin-films solar cells.

As shown in Fig. 5(b) the transmittance of the Cr-alloy is negligible for thicknesses $w$ larger than $1 \mu \mathrm{m}$, whereas for the Ti-compound (Fig. 5(a)) it is almost restricted to the interval of forbidden absorption (0.5-1 eV). In view of these results, $w$ is a parameter of which optimization should be taken into account, because on the one hand by means of the intermediate band, we achieve an enhancement of the absorption capabilities, but on the other hand if we do not take into account the optimum width of the sample, the absorptivity can be reduced and part of the effect of the intermediate band can be lost. This makes it necessary to find a compromise between the absorption coefficient and the thickness of the absorber to be used for photovoltaic purposes. 


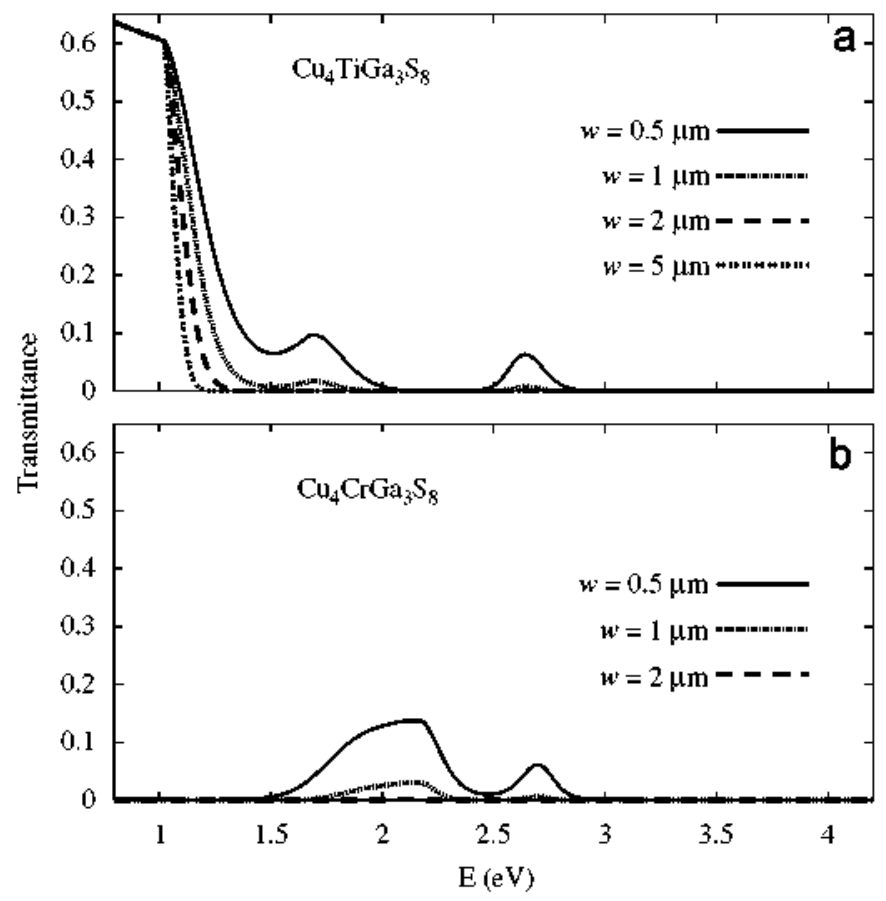

Fig. 5. (Color online) Transmittance of the intermediate-band materials as a function of the width of the sample $w$ : (a) $\mathrm{Cu}_{4} \mathrm{TiGa}_{3} \mathrm{~S}_{8}$ and (b) $\mathrm{Cu}_{4} \mathrm{CrGa}_{3} \mathrm{~S}_{8}$.

\section{Conclusions}

In this work, we have considered interband and direct transitions for the prediction of the best substituent for $\mathrm{CuGaS}_{2}$ in the quest of an efficient intermediate-band material, by means of the enhancement of their optical absorption.

Ti and $\mathrm{Cr}$-substituted $\mathrm{CuGaS}_{2}$ present a particular electronic structure due to the $3 \mathrm{~d}$ valence electrons of the transition metal that form the intermediate band, which produces a significant enhancement in the absorption coefficient of the alloys. As a consequence of these characteristics, we propose $\mathrm{Ti}$ and $\mathrm{Cr}$-substituted $\mathrm{CuGaS}_{2}$ as the best chalcopyrite-type candidates, absorbing across the full solar spectrum range, for intermediateband solar cells. We have studied their reflectance and transmittance and concluded that the thickness of the material to be used as an absorber in a cell should be optimized in order not to lose part of the effect of the intermediate band.

Due to the position of the partially filled intermediate band inside the gap and because of the features of the absorption coefficient of the derived alloy, we consider Ti as the optimum substituent of $\mathrm{Ga}$ in $\mathrm{CuGaS}_{2}$ for photovoltaic purposes.

Experimental preparations of this material have been initiated and we believe that this work will help in the interpretation and understanding of future experiments.

\section{Acknowledgments}

This work was supported by the CALIBAND Project (MAT200610618) and the Consolider Ingenio 2010 Program, through the Project GENESIS-FV (Ref. CSD2006-04) of The Spanish Ministry of Education and Science and the Community of Madrid NUMANCIA Project (S-05050/ENE/0310). The computer resources provided by the Madrid Supercomputing Center (CeSViMa) are acknowledged. l.A. would also like to acknowledge the MEC for a FPU grant.

\section{References}

[1] P. Palaciós, 1. Aguilera, K. Sánchez, J. C. Conesa, P. Wahn' on, Transition-MetalSubstituted Indium Thiospinels as Novel Intermediate- Band Materials: Prediction and Understanding of Their Electronic Properties, Phys, Rev, Lett. 101 (2008) 046403(4pp).

[2] A. Luque, A. Martí, Increasing the efficiency of ideal solar cells by photon induced transitions at intermediate levels. Phys. Rev. Lett. 78 (1997) 5014-5017.

[3] R. Lucena, I. Aguilera, P. Palacios, P. Walmón, J.C. Conesa, Synthesis and spectral properties of nanocrystalline $V$-substituted $I_{2} S_{3}$, a novel material for more efficient use of solar sadiation, Chem. Mater. 20 (2008) 5125-5127.

[4] P. Palacios, K. Sánchez, J.C. Conesa, P. Wahnón, First principles calculation of isolated intermediate bands formation in a transition metal-doped chalcopyrite-type semiconductor. Plyys. Status 5olidi A 203 (2006) 1395-1401.

[5] P. Palaciós, K. Sánchez, J.C. Conesa, J.J. Femández, P. Wahnón, Theoretical modelling of intermediate band solar cell materials based on metal-doped claalcopyrite compounds, Thin Solid Films 515 (2007) 6280-6284.

[6] P. Palacios, K. Sánchez, P. Wahnón, J.C. Conesa, Characterization by ab-initio calculations of intermediate band material based on chalcopvrite semiconductors substituted with several transition metals, J. Solar Energy Eng. 129 (2007) 314-318.

[7] S. Picozzi, Y.-J. Zhao. A. J. Freeman, B. Delley, Mn-doped $\mathrm{CuGa5}_{2}$ chalcopyrites: An ab initio study of ferromagnetic semiconductors, Phys. Rev. B 66 (2002) 205206(6pp).

[8] Y.-J. Zhao, A. Zunger, Electronic structure and ferromagnetism of $\mathrm{Mn}$ substituted CuAlS , CuGaS, CulnS 2 , CuGaSe, and CuGaTe 2 , Pliys. Rev, B 69. (2004) $104422(8 \mathrm{pp})$.

[9] P. Prabukanthan R. Dhanasekaran, Influence of Mn doping on CuGaS single crystals grown by CVT method and their characterization. J. Phys. D Appl. Phys. 41, (2008) 115102(6pp).

[10] M.J. Alonso, K. Wakita, J. Pascual, M. Garriga, N. Yamamoto, Optical functions and electronic structure of CulnSe ${ }_{2}$ CuGaSe, CulnS $S_{2}$, and $\mathrm{CuGaS}_{2}$, Phys. Rev. B 63, (2001) 075203(13pp).

[11] J.R. Botha, M.S. Branch, P.R. Berndt, A.W.R. leitch, J. Weber, Defect chemistry in $\mathrm{CuGaS}_{2}$ thin films: a photoluminescence study. Thin Solid Films 515 (2007) $6246-6251$.

[12] C. Bellabarba, J. González, C. Rincón, Optical-absorption spectrum near the exciton band edge in CuGaS $\mathrm{C}_{2}$ at $5 \mathrm{~K}$, Phys. Rev. B 53 (1996) 7792-7796.

[13] J.E. Jaffe, $A$. Zunger. Theory of the band-gap anomaly in $\mathrm{ABC}_{2}$ chalcopyrite semiconductor's. Pliys. Rev, B 29 (1984) 1882-1906.

[14] S. Laksari, A. Chahed, N. Abbouni, O. Benhelal, B. Abbar, First-principles calculations of the structural, electronic and optical properties of $\mathrm{CuGa}_{2}$ and $\mathrm{AgGaS}_{2}$, Comput. Mater. Sci. 38 (2006) 223-230.

[15] J.L. Shay. B. Tell, H.M. Kasper, L.M. Schiavone, p-d Hybridization of the valence bands of I-lll- $V_{2}$ compounds, Phys. Rev. B 5 (1972) 5003-5005.

[16] M.S. Branch, P.R. Berndt, J.R. Botha, A.W.R. Leitch, J. Weber, Structure and morphology of CuGaS, thin films. Thin Solid Films 431-432 (2003) 94-98.

[17] B. Tell, J.L. Shay, H.M. Kasper, Electrical properties, optical properties, and band structure of $\mathrm{CuCaS}_{2}$ and $\mathrm{CulnS}_{2}$. Phys. Rev. B 4 (1971) 2463-2471.

[18] G. Kresse, J. Hafner, $A b$ initio molecular dynamics for liquid metals, Phys. Rev. B 47 (1993) 558-561.

[19] G. Kresse, J. Furthmüller. Efficient iterative schemes for ab initio totalenergy calculations using a plane-wave basis set, Phys. Rev. B 54 (1996) 11169-11186.

[20] G. Kresse, D. Joubert, From ultrasoft pseudopotentials to the projector augmented-wave method, Phys. Rev. B 59 (1999) 1758-1775.

[21] J.P. Perdew. J.A. Chevary, 5.H. Vosko, K.A. Jackson, M.R. Pederson, D.J. Singl, C. Fiolhais, Atoms, molecules, solids, and surfaces: applications of the generalized gradient approximation for exchange and correlation, Phys. Rev. B 46 (1992) 6671-6687.

[22] M. Gajdos, K. Hummer, G. Kresse, ]. Furtḧ̈ller, F. Bechstedt, linear optical properties in the projector-augmented wave methodology. Phys. Rev. B 73. (2006) 045112 (9pp).

[23] G. Kresse, D. Joubert, Structure and morphology of CuGas $\mathrm{C}_{2}$ thin films, Phys. Rev. B 59 (1999) 1758-1775.

[24] P.E. Blöchl, Projector augmented-wave method, Phys. Rev. B 50 (1994) 17953-17979.

[25] J.]. Fernández, C. Tablero, P. Wahnón, Development and implementation of the exact exchange method for semiconductors using a localized basis set, Comput. Mater. 5 ci. 28 (2003) 274-286.

[26] L. Hedin, New method for calculating the one-particle Green's function with application to the electron-gas problem. Phys. Rev. 139 (1965) A796-A823.

[27] M.S. Hybertsen, S.G. Louie, First-principles theory of quasiparticles: calculation of band gaps in semiconductors and insulator's. Phys. Rev, lett. 55 (1985) 1418-1421.

[28] P. Wahnón, P. Palacios, K. Sánchez, J. Aguilera, J.C. Conesa, Engineering intermediate band materials based on metal-doped chalcogenides compounds by quantum mechanical calculations, in: G. Willeke, H. Ossenbrink, P. Helm (Eds.), Proceedings of the 22nd European Photovoltaics Solar Energy Conference, WJP-Renewable Energies, Munich, 2007, p. 56-59, ISBN-3936338-22-1.

[29] ]. Aguilera, P. Palacios, P. Walnón, Optical properties of chalcopyrite-type intermediate transition metal band materials from first principles. Thin Solid Films 516 (2008) 7055-7059. 